\title{
A Sub-6 GHz Vital Signs Sensor Using Software Defined Radios ${ }^{+}$
}

\author{
Onur Toker* and Rawa Adla \\ Department of ECE, Florida Polytechnic University, Lakeland, FL 33805, USA; e-mail@e-mail.co \\ * Correspondence: otoker@floridapoly.edu \\ + Presented at the 7th International Electronic Conference on Sensors and Applications, 15-30 November 2020; \\ Available online: https:/ /ecsa-7.sciforum.net/.
}

Published: 15 November 2020

\begin{abstract}
Recently, there is a big demand on contactless devices for health safety, therefore developing a low-cost contactless breathing sensor will have a great benefit for many patients and healthcare workers. In this paper, we propose a contactless sub- $6 \mathrm{GHz}$ breathing sensor with an implementation using a low-cost USRP device B205-mini. A detailed performance analysis of the proposed system with different sensor algorithms is presented. The proposed system estimates the channel phase shift and detects the presence of low frequency oscillations in the estimated phase shift. Compared to $24 \mathrm{GHz}$ or $77 \mathrm{GHz}$ FMCW radar based systems using distance measurements, the proposed system is simpler, can be built by using more economical RF components, and requires lower sampling frequencies. Another key advantage of the proposed system is that, even a very narrow unused frequency band is enough for the operation of the sensor. When operated at frequencies shared by other devices, the proposed system can turn off the transmitter at randomly selected intervals to detect the presence of other transmission activities, and then can switch to a different operating frequency. We provide both Python and Octave/Matlab based implementations which are available in a public GitHub repo.
\end{abstract}

Keywords: Contactless sensors; Vital signs sensors; RF sensors

\section{Introduction}

Contactless vital signs detection, and measurement is important problem for nursing homes and hospitals. There are various published papers using different kinds of radar techniques for contactless measurements, as well as optical techniques which are contact based, see [1] and references therein. In this paper, we propose a low-cost channel phase shift estimation based technique which can be easily implemented using commerical of the shelf components and software defined radio (SDR) devices, more specifically Universal Software Radio Peripheral (USRP) of type B205-mini.

Use of SDR and USRP devices for prototying radar systems is known for a long time, see [2,3] and references therein. In [4], a wireless condition monitoring design is presented using microelectromechanical system (MEMS) sensors, in [5], a continuous-wave (CW) radar based system is proposed for contactless monitoring, and in [6], another contactless microwave sensor is proposed for detection of vibrations. In [7], a digital signal processor system is proposed for Doppler radar sensing of vital signs. In [8], a multi-antenna based contactless radar system is proposed for assessment of human respiration patterns. In $[9,10]$, two different $77 \mathrm{GHz}$ systems are proposed for remote monitoring of vitals signs. Finally, [11] has a sub- $6 \mathrm{GHz}$ based noncontact based vital signs sensor. Contrary to many of reported systems, our proposed system is not based on distance measurements. It is based on channel phase shift estimation, and does not require any kind of frequency sweep which simplifies the RF subsystem considerably. 
This paper is organized as follows: In Section 2, we describe the experimental setup, and in Section 3, we present the implementation details. In Section 4, proposed features are presented with detailed analysis of the performance of single feature based classifiers. Finally, we make some concluding remarks in Section 5.

\section{Experimental Setup}

Our experimental setup is shown in Figure 1: There is laptop computer running Linux, a USRP-B205 mini, and two coffee can antennas. USRP-B205 mini is a sub-6 GHz device, and has a tuneable phase-locked loop (PLL) controlled local oscillator (LO). In this paper, we set the LO frequency to $3 \mathrm{GHz}$, however the $\mathrm{LO}$ frequency is a design paramater which will be investigated in a future version of the paper.

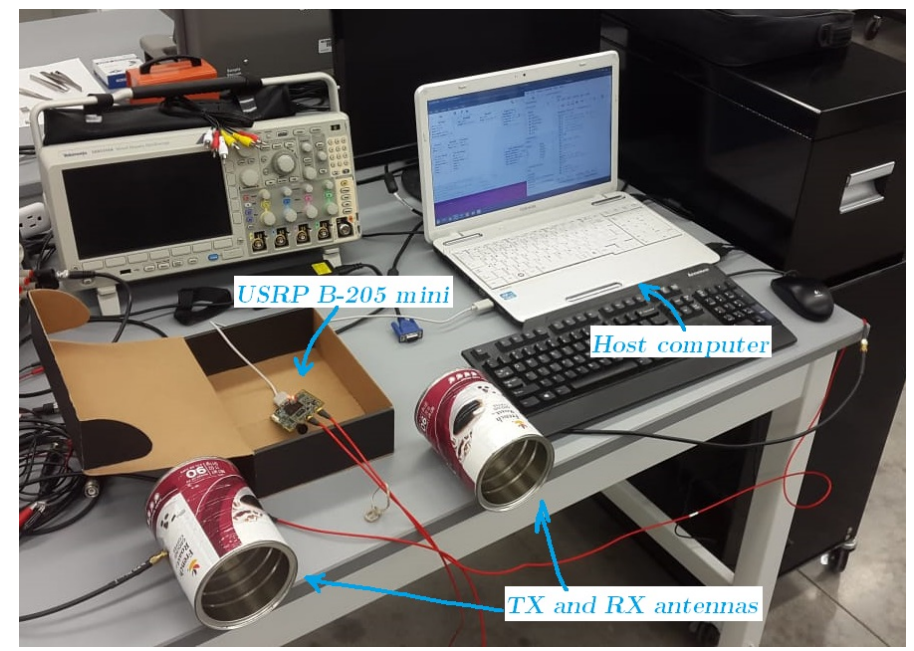

Figure 1. Sub-6 GHz vital signs detector using a USRP B205-mini.

The high level system block diagram is shown in Figure 2. Basically, on the transmitter side the signal $x_{b}(t)$ is chosen as a complex sinusoid of frequency approximately $20 \mathrm{kHz}$, and on the receiver side $y_{b}(t)$ is the received baseband signal. The proposed vital signs detector estimates the phase shift between $x_{b}(t)$ and $y_{b}(t)$ at a rate of 50 times per second, which is also a configurable design parameter. The baseband sampling frequency is set as $4 \mathrm{MHz}$, and is two orders of magnitude larger than the baseband analog signal frequency of $20 \mathrm{kHz}$.

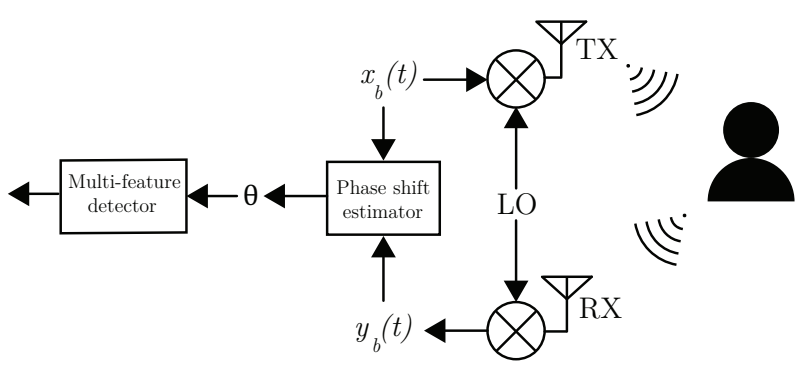

Figure 2. High level block diagram of the proposed system.

The phase shift, $\theta$, is estimated by comparing the peak values of FFT's of $x_{b}(t)$ and $y_{b}(t)$, more precisely by division and conversion to polar format. Estimated phase shift values are then fed to a single feature based detector system. Basically, we propose the use of two different features and linear classifiers for vital signs detection. Estimation of vital parameters, like the breating rate, are not addressed and will be investigated in a future version of the paper. In this paper, we focus on the binary classification problem for vital signs detection. 


\section{Implementation Details}

Authors developed two different software packages for this research, which is available at the public GitHub repo https: / / github.com/onurtoker/vitalSigns.

\subsection{Python + UHD Driver Based Implementation}

Our first approach is based on the UHD driver, and Python, and does not use any GNU Radio package or MATLAB/Octave like high level tools. Compared to a GNU radio and MATLAB/Octave based approach, it may be less user friendly, but it can show the estimated phase shift in a scope like window in real-time. The current implementation available in the GitHub repo does not have a specific real-time feature extraction and classification method coded, however all of these can be added easily by using numpy and scipy libraries. For the research results presented in this paper, we first recorded data, i.e., $\theta$ values, and then tested different features and classifiers using an offline approach. In Table 1, we summarize the version numbers of the major software components used in the Python + UHD driver based approach.

Table 1. Software versions.

\begin{tabular}{ll}
\hline Ubuntu & 18.04 .1 \\
PyCharm & 2020.1 \\
Python & 3.6 .9 \\
numpy & 1.16 .3 \\
scipy & 1.2 .1 \\
\hline
\end{tabular}

\subsection{GNU Radio + Octave Implementation}

Our second approach is based on higher level and more user friendly tools: GNU radio and Octave. For the data transfer between a GNU radio graph and Octave script, we used the ZMQ library which is a standard high-performance asynchronous messaging library. The GNU radio graph used for this experiment is shown in Figure 3. The default transmitter frequency is $2.4 \mathrm{GHz}$, but we did all experiments at $3 \mathrm{GHz}$. The optional XMLRPC block of the GNU radio allows the Octave script to the change GNU radio graph parameters while the graph is running.

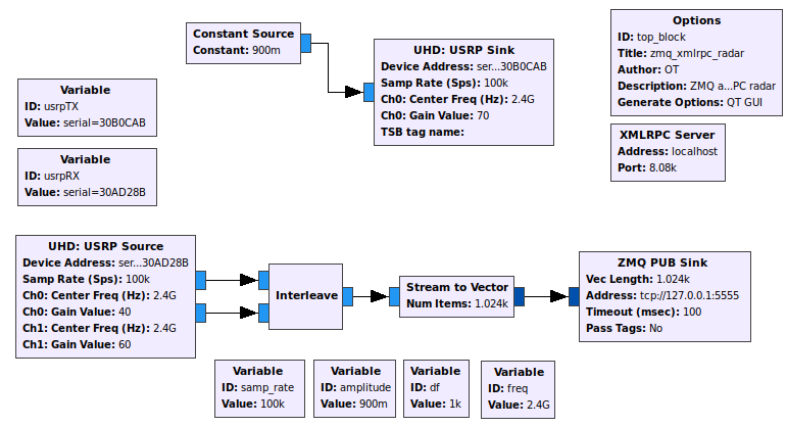

Figure 3. GNU radio graph with default parameters.

\section{Proposed Features and Results}

In this section, we will summarize the proposed features and the corresponding linear classifiers for vital signs detection. In Figure 4 on the top, we have phase shift plots for 3 different positive cases, i.e., when there is breathing activity in front of the sensor (Class 1). Plots shown at the bottom of Figure 4 are for 3 different negative cases, i.e., when there is no motion or breating activity around the sensor (Class 0). To be able to illustrate all data in fewer number of plots, we first remove the DC bias, and then level shift all by a different amount to make sure that phase plots do not overlap. As it is 
clear from Figure 4, phase plots for the positive cases (Class 1) look quite different compared to the negative cases (Class 0$)$.
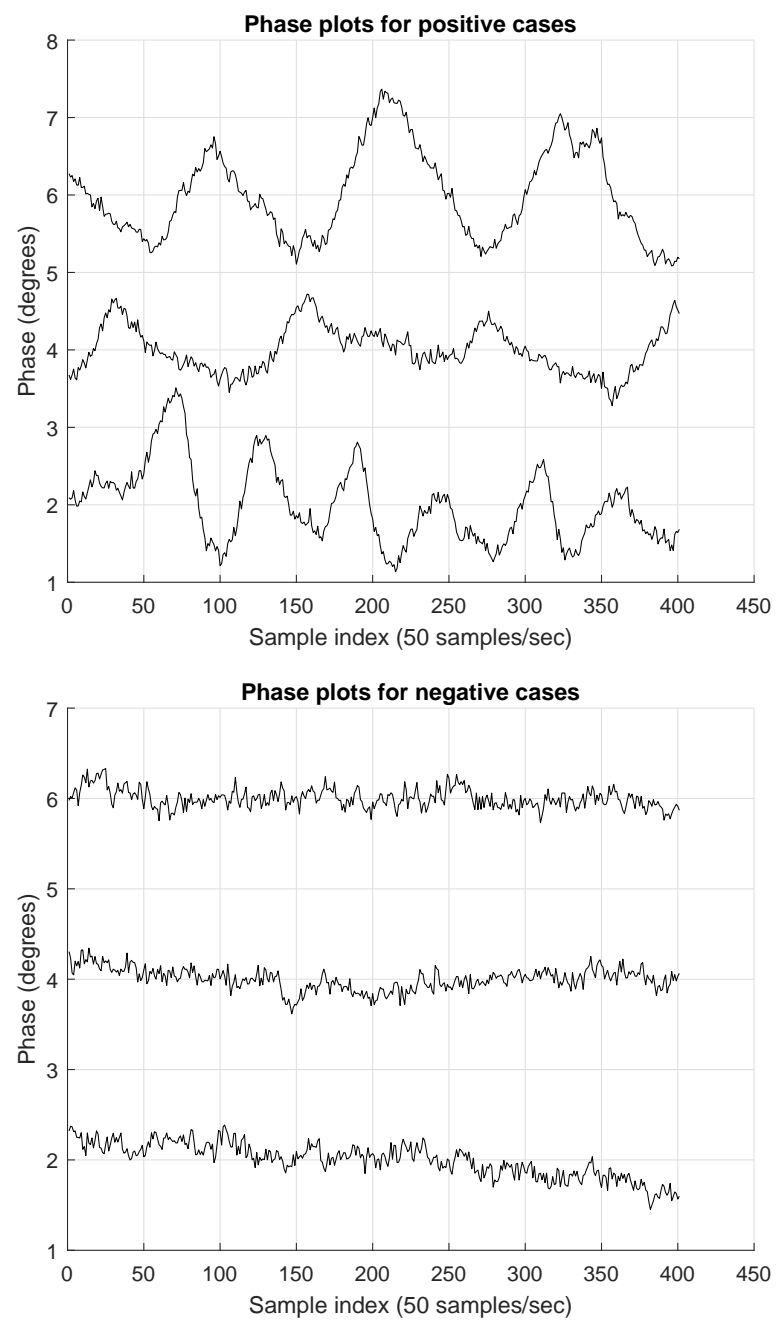

Figure 4. Phase plots for 3 positive (top) and 3 negative cases (bottom). All after DC bias removal and some level shifting.

\subsection{Feature 1 (Local Variance)}

Our first proposed feature is the variance of local $N_{c}$ phase shift values, or in a real-time setting variance of the latest $N_{c}$ phase shift measurements. We have chosen $N_{c}=50$, and $N_{c}=150$, and computed the variance of local/past $N_{c}$ samples. In Figure 5, the variance is plotted as a function of the sample index.

In Figure 6, histogram confusion plots for $N_{c}=50$ and $N_{c}=150$ are shown. The overlap is smaller less for $N_{c}=150$, however a design based on $N_{c}=50$ uses measurements aquired within $1 \mathrm{~s}$, whereas $N_{c}=150$ corresponds to $3 \mathrm{~s}$. Hence, although $N_{c}=150$ results a more reliable detector, in the event of loss of vital signs, it is expected to respond after a longer delay compared to $N_{c}=50$. Both reliability as well as the response time in the event of loss of vitals signs are critical performance metrics.

The false alarm is defined as the detector output being "Class 0 " although there are still vital signs, and the miss is defined as the detector output being "Class 1" although there are no vital signs. The optimal classifier will be the detector which minimizes the sum of $P_{F}$, the false alarm rate, and $P_{M}$, the probability of miss. More precisely, it will be

If "Feature 1 " $\geq \gamma$, then classify as Class 1 , 


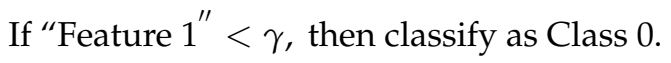

where $\gamma=0.00200$ and 0.00235 for $N_{c}=50$ and 150 respectively. The value of $\gamma$ is estimated as the $x$-axis of the intersection of two Gaussian distrubitions in Figure 6.
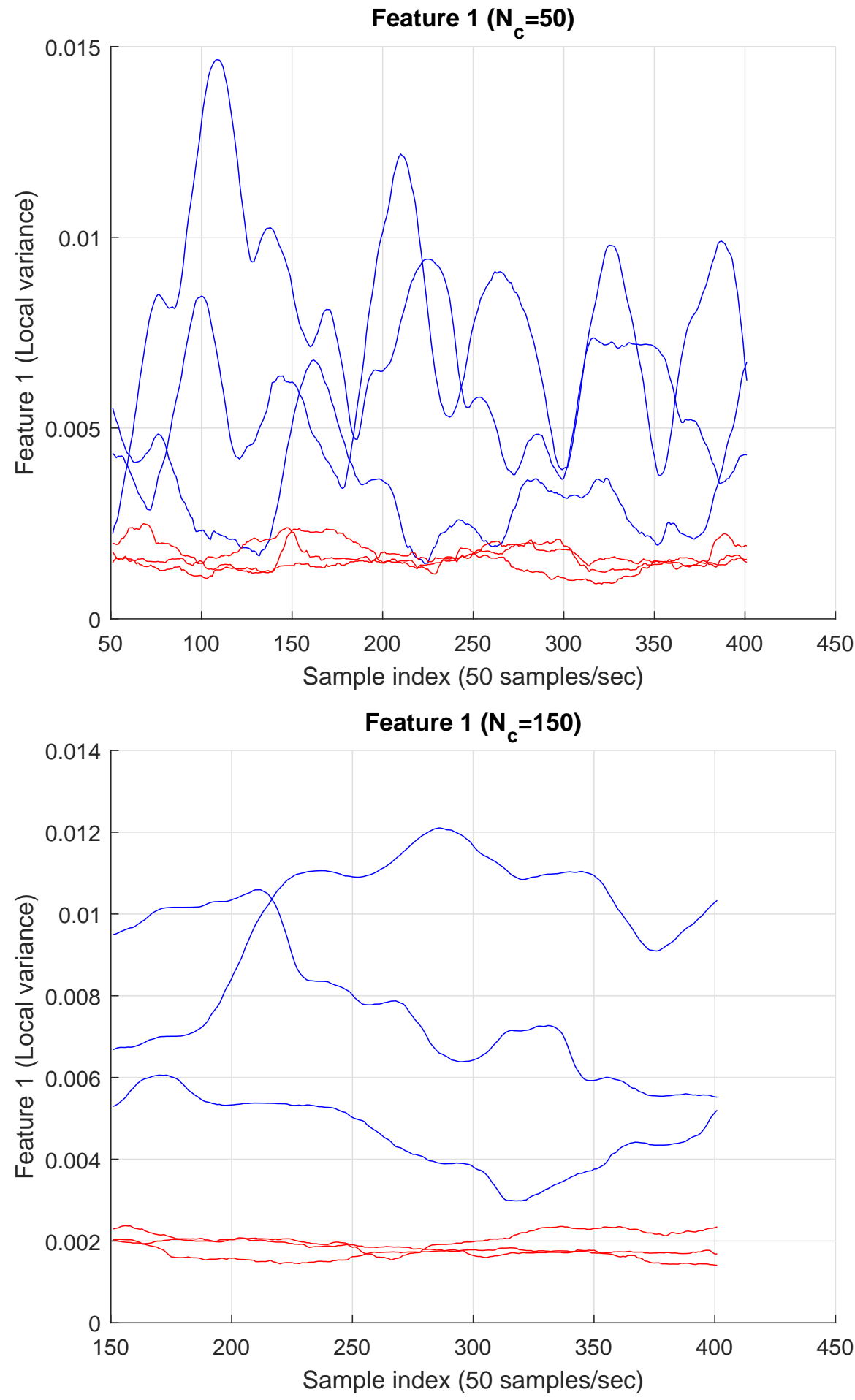

Figure 5. Feature 1: Blue is for Class 1 , and red is for Class 0 . On the top $N_{c}=50$, and at the bottom $N_{c}=150$. 

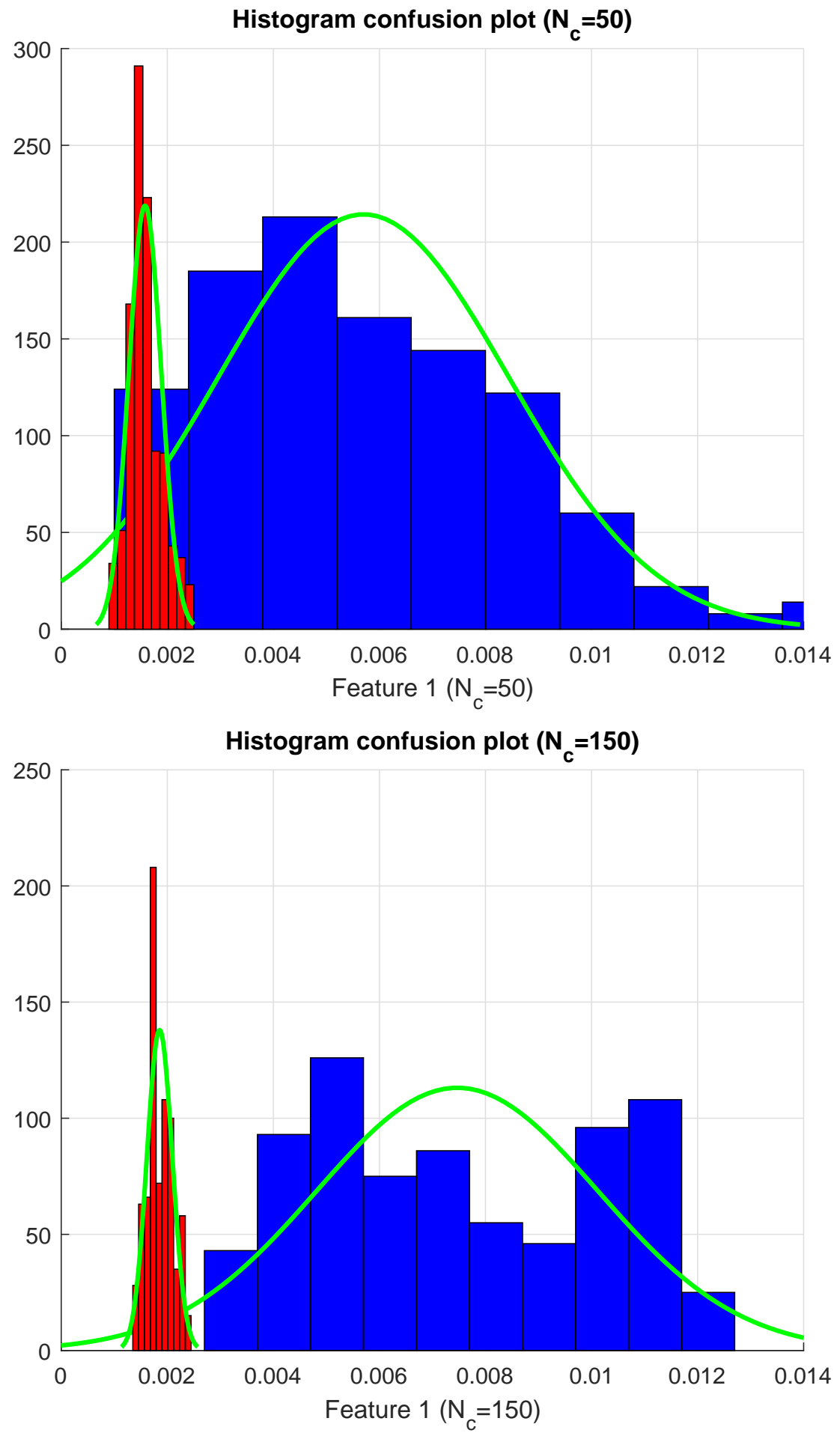

Figure 6. Blue is for Class 1, red is for Class 0, and green are fitted Gaussian distributions. On the top $N_{c}=50$, and at the bottom $N_{c}=150$.

\subsection{Feature 2 (Ratio of Low Frequency Energy Content)}

Our second feature is the percentage of energy (of the estimated phase shift signal, $\theta$ ) which lies between $0.35 \mathrm{~Hz}$ and $1.5 \mathrm{~Hz}$. Note that the DC bias is always removed, and the end points of the band $0.35-1.5 \mathrm{~Hz}$ are design parameters that can be varied for further optimization.

In Figure 7, FFT plots for a positive and a negative case are shown, and it is clear that for the positive case the frequency band $[0.35,1.5] \mathrm{Hz}$ has much higher relative energy content compared to 
the negative case. Furthermore, the frequency at which the peak is observed can be used to estimate the breating rate as well.

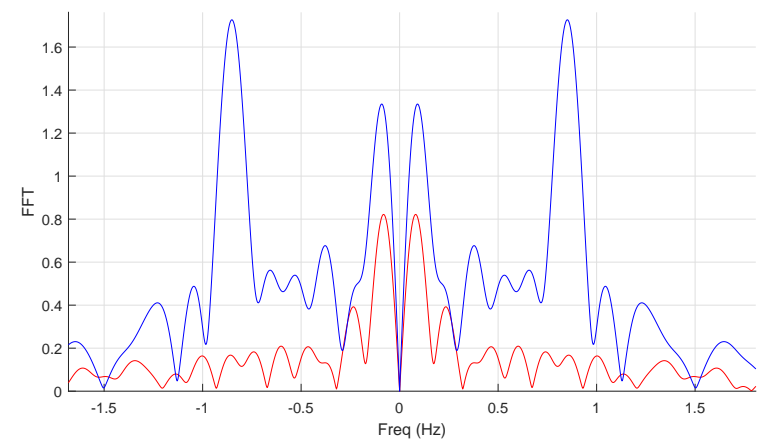

Figure 7. FFT amplitude plots vs frequency: Red for class 0, and blue for class 1.

Basically, we define our second feature as

$$
\text { “Feature } 2^{\prime \prime}=\frac{\text { Energy of } \theta(t) \text { in } 0.35 \mathrm{~Hz}-1.5 \mathrm{~Hz}}{\text { Total energy of } \theta(t)}
$$

and according to this definition, we get the values shown in Table 2.

Table 2. Feature 2 (Energy ratios).

\begin{tabular}{ccc}
\hline Experiment No. & Class Type & "Feature 2" \\
\hline 1 & Class 0 & 0.3699 \\
2 & Class 0 & 0.4555 \\
3 & Class 0 & 0.5833 \\
4 & Class 1 & 0.8067 \\
5 & Class 1 & 0.8354 \\
6 & Class 1 & 0.9283 \\
\hline
\end{tabular}

In Figure 8 , "Feature 2" values are shown on a 1-D axis, which exhibits a very clear separation between two classes.

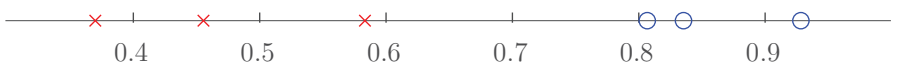

Figure 8. Feature 2: Red for class 0, and blue for class 1 .

Our "Feature 2" based classifier is

$$
\begin{aligned}
& \text { If "Feature2" } \geq \gamma \text {, then classify as Class } 1, \\
& \text { If "Feature2" }<\gamma \text {, then classify as Class } 0 .
\end{aligned}
$$

where $\gamma$ is selected the average of class means, i.e., $\gamma \approx 0.65$.

For this feature, histograms do not overlap and both distributions are well seperated from each other. This is clearly better than the first feature, however computationally it is more demanding. If a mobile low-power device is intented to be designed for vital signs monitoring, computational load and associated increase in the power consumption may be a concern.

\section{Conclusions}

In this paper, we have presented a sub-6 GHz vital signs detector system and experimentally analyzed its performance. We considered only the binary classification problem, and proposed two 
different features. Estimation of vital paramaters is not addressed, and will be considered in a future version of the paper. Sensor fusion using both of these features will also be addressed in a future version. Overall, use of the first or the second of proposed feature results an effective and low-cost vital signs detection system. For both features, the histogram confusion plots imply low rate of false positive and negatives.

Author Contributions: Both authors contributed to all parts equally. All authors have read and agreed to the published version of the manuscript.

Funding: This work has been supported in part by Florida Polytechnic University grant GR-1900022, Advanced Mobility Institute grants GR-2000028, GR2000029, and the NSF grant 1919855. Both authors acknowledge their individual startup funds.

Conflicts of Interest: The authors declare no conflict of interest. The funders had no role in the design of the study; in the collection, analyses, or interpretation of data; in the writing of the manuscript, or in the decision to publish the results.

\section{References}

1. Raffo, A.; Costanzo, S.; Massa, G.D. Software Defined Doppler Radar as a Contactless Multipurpose Microwave Sensor for Vibrations Monitoring. Sensors 2017, 17, 115 doi:10.3390/s17010115.

2. Braun, M. OFDM Radar Algorithms in Mobile Communication Networks. PhD Thesis, Karlsruher Instituts für Technologie, Karlsruhe, Germany, 2014. Available online: https:/ / publikationen.bibliothek.kit.edu/ $1000038892 / 2987095$ (accessed on)

3. Prager, S.; Thrivikraman, T.; Haynes, M.S.; Stang, J.; Hawkins, D.; Moghaddam, M. Ultrawideband Synthesis for High-Range-Resolution Software-Defined Radar. IEEE Trans. Instrum. Meas. 2020, 69, 3789-3803. doi:10.1109/TIM.2019.2937423.

4. Vogl, A.; Wang, D.T.; Storås, P.; Bakke, T.; Taklo, M.M.; Thomson, A.; Balgård, L. Design, process and characterisation of a high-performance vibration sensor for wireless condition monitoring. Sens. Actuators $A$ Phys. 2009, 153, 155-161. doi:10.1016/j.sna.2009.04.011.

5. Pieraccini, M.; Fratini, M.; Parrini, F.; Macaluso, G.; Atzeni, C. High-speed CW step-frequency coherent radar for dynamic monitoring of civil engineering structures. Electron. Lett. 2004, 40, 907-908. doi:10.1049/el:20040549.

6. Grazzini, G.; Pieraccini, M.; Dei, D.; Atzeni, C. Simple microwave sensor for remote detection of structural vibration. Electron. Lett. 2009, 45, 567-569. doi:10.1049/el.2009.1107.

7. Lohman, B.; Boric-Lubecke, O.; Lubecke, V.M.; Ong, P.W.; Sondhi, M.M. A digital signal processor for Doppler radar sensing of vital signs. IEEE Eng. Med. Biol. Mag. 2002, 21, 161-164. doi:10.1109/MEMB.2002.1044188.

8. Gu, C. Assessment of Human Respiration Patterns via Noncontact Sensing Using Doppler Multi-Radar System. Sensors 2015, 15, 6383-6398. doi:10.3390/s150306383.

9. Wang, Y.; Wang, W.; Zhou, M.; Ren, A.; Tian, Z. Assessment of Human Respiration Patterns via Noncontact Sensing Using Doppler Multi-Radar System. Sensors 2020, 20, 2999. doi:10.3390/s20102999.

10. Alizadeh, M.; Shaker, G.; Almeida, J.C.M.D.; Morita, P.P.; Safavi-Naeini, S. Remote Monitoring of Human Vital Signs Using mm-Wave FMCW Radar. IEEE Access 2019, 7, 54958-54968. doi:10.1109/ACCESS.2019.2912956.

11. Park, J.; Hong, Y.; Lee, H.; Jang, C.; Yun, G.; Lee, H.; Yook, J. Noncontact RF Vital Sign Sensor for Continuous Monitoring of Driver Status. IEEE Trans. Boimedical Circuits Syst. 2019, 13, 493-502. doi:10.1109/TBCAS.2019.2908198.

Publisher's Note: MDPI stays neutral with regard to jurisdictional claims in published maps and institutional affiliations.

(C) 2020 by the authors. Licensee MDPI, Basel, Switzerland. This article is an open access article distributed under the terms and conditions of the Creative Commons Attribution (CC BY) license (http:// creativecommons.org/licenses/by/4.0/). 